\title{
Cutibacterium acnes (formerly Propionibacterium acnes) isolated from prosthetic joint infections is less susceptible to oxacillin than to benzylpenicillin
}

\author{
Sara Ridberg${ }^{1}$, Bengt Hellmark ${ }^{1}$, Åsa Nilsdotter ${ }^{2}$, Bo Söderquist ${ }^{1 凶}$ \\ 1. School of Medical Sciences, Faculty of Medicine and Health, Örebro University, Örebro, Sweden \\ 2. Department of Infectious Diseases and Department of Clinical and Experimental Medicine, Linköping University, Norrköping, Sweden \\ $\square$ Corresponding author: Bo Söderquist, MD, PhD, Professor Department of Laboratory Medicine, Clinical Microbiology, Örebro University Hospital, SE-701 \\ 85 Örebro, Sweden
}

(c) The authors. This is an open access article distributed under the terms of the Creative Commons Attribution (CC BY) license (https://creativecommons.org/licenses/ by/4.0/). See http://ivyspring.com/terms for full terms and conditions.

Received: 2018.10.25; Accepted: 2019.03.12; Published: 2019.04.20

\begin{abstract}
Introduction: The frequency of prosthetic joint infections (PJIs) due to Cutibacterium acnes (formerly Propionibacterium acnes) is increasing, especially shoulder PJls. The recommended antibiotic prophylaxis for hip and knee arthroplasties is beta-lactam antibiotics, predominantly cephalosporins. However, for example in Sweden, isoxazolyl-penicillin cloxacillin is used. No specific recommendations for shoulder arthroplasties are available. The aim of the present study was to determine the minimum inhibitory concentration (MIC) values for different antibiotics for $C$. acnes; and, more specifically, to compare the MIC values for benzylpenicillin and oxacillin.
\end{abstract}

Materials and methods: Minimum inhibitory concentration (MIC) values for nine different antibiotic agents were obtained by gradient test (Etest) using strains of $C$. acnes $(n=57)$ isolated from PJls from shoulders $(n=31)$, hips $(n=21)$, and knees $(n=5)$.

Results: All isolates had low MIC values for most of the tested antibiotic agents, and showed a wild type MIC distribution. The exception was clindamycin with $9 \%$ of the isolates displaying decreased susceptibility. The MIC values obtained for benzylpenicillin were significantly lower than the MIC values for isoxazolyl-penicillin (oxacillin).

Conclusion: These in vitro results indicate that benzylpenicillin might be a more effective prophylactic treatment to prevent shoulder PJls caused by C. acnes. However, further studies on the subject are needed, and the effectiveness of the prophylactic treatment should be evaluated using randomized controlled studies and/or register-based studies.

Key words: Cutibacterium acnes, Propionibacterium acnes, prosthetic joint infections, antibiotic susceptibility testing, antibiotic prophylaxis

\section{Introduction}

Infections associated with orthopedic joint implants, such as prosthetic joint infections (PJIs), are a major threat to patients' quality of life following prosthetic joint surgery. Perioperative antibiotic prophylaxis is universally used to reduce the risk of surgical site infections [1]. In many countries, various cephalosporins are used as first-line prophylaxis [2]. However, in Sweden, isoxazolyl-penicillin cloxacillin is recommended as antibiotic prophylaxis for all primary joint replacements [PRISS Expert Group 2; http://lof.se/patientsakerhet/vara-projekt/rekomm endationer/].

The most common microorganisms that cause PJIs are staphylococci [2,3]. Anaerobic bacteria, predominantly Cutibacterium acnes (formerly Propionibacterium acnes), cause less than $5 \%$ of PJIs $[4,5,6,7]$. However, $C$. acnes has been reported to be responsible for $>50 \%$ of infections following shoulder surgery 
$[7,8]$. Therefore, at centers for shoulder surgery in Sweden, the recommended antibiotic prophylaxis regimens have been reviewed. Cloxacillin was replaced by clindamycin or supplemented by adding benzylpenicillin (personal communication).

The aim of the present study was to determine the minimum inhibitory concentration (MIC) values for different antibiotics against $C$. acnes isolated from patients with PJIs; and, more specifically, to compare the MIC values for benzylpenicillin and oxacillin in order to determine if a change in the existing antibiotic prophylactic regimen for shoulder arthroplasties is justified.

\section{Materials and Methods}

C. acnes $(\mathrm{n}=57)$ isolated from orthopedic implant-associated infections mainly PJIs were obtained from the Departments of Clinical Microbiology at the University Hospitals of Örebro and Linköping from 2002 to 2015. Isolates came from shoulder $(n=31)$, hip $(n=21)$, and knee $(n=5)$ orthopedic implant-associated infections.

The antibiotic susceptibility pattern was tested for nine different antibiotics; benzylpenicillin, oxacillin, ampicillin, amoxicillin, cefuroxime, clindamycin, rifampicin, daptomycin, and vancomycin. MIC values were determined by Etest (bioMérieux, Marcy -l'Étoile, France). The in vitro antibiotic susceptibility testing was performed on FAA plates $(4.6 \%$ LAB 90 fastidious anaerobe agar; LAB M, Heywood, United Kingdom, supplemented with 5\% (vol/vol) horse blood) with 0.5 McFarland suspensions of bacteria in $\mathrm{NaCl}$ and incubation at $36^{\circ} \mathrm{C}$ under anaerobic conditions for 1 day.

\section{Ethics}

This study used bacterial isolates from humans. No tissue material or other biological material was stored from the patients. All information regarding these isolates was anonymized.

\section{Results}

The distributions of MIC values for the nine antimicrobial agents are presented in Figures 1a-i. In general, the MIC values for the beta-lactam antibiotics were low and showed a wild type MIC distribution pattern. The lowest MIC values were noted for benzylpenicillin, with a $\mathrm{MIC}_{50}$ of $0.008 \mathrm{mg} / \mathrm{L}$ and a $\mathrm{MIC}_{90}$ of $0.012 \mathrm{mg} / \mathrm{L}$. The $\mathrm{MIC}_{50}$ and $\mathrm{MIC}_{90}$ values for cefuroxime were $0.023 \mathrm{mg} / \mathrm{L}$ and $0.047 \mathrm{mg} / \mathrm{L}$, respectively.

The MIC values for clindamycin deviated from the wild type group, and 5/57 (8.8\%) of the isolates were resistant. An MIC value of $>256 \mathrm{mg} / \mathrm{L}$ was noted for $3 / 57(5.3 \%)$ of the isolates, indicating high-level resistance to clindamycin. The MIC values for rifampicin were low, with an $\mathrm{MIC}_{50}$ of $0.003 \mathrm{mg} / \mathrm{L}$, an $\mathrm{MIC}_{90}$ of $0.006 \mathrm{mg} / \mathrm{L}$, and none of the isolates displayed decreased susceptibility. Daptomycin had an $\mathrm{MIC}_{50}$ of $0.38 \mathrm{mg} / \mathrm{L}$ and an $\mathrm{MIC}_{90}$ of $0.5 \mathrm{mg} / \mathrm{L}$, while vancomycin had an $\mathrm{MIC}_{50}$ of $0.19 \mathrm{mg} / \mathrm{L}$ and an $\mathrm{MIC}_{90}$ of $0.25 \mathrm{mg} / \mathrm{L}$.

Figure 2 provides a comparison of the MIC values for oxacillin and benzylpenicillin. For oxacillin, the $\mathrm{MIC}_{50}$ was $0.125 \mathrm{mg} / \mathrm{L}$ and the $\mathrm{MIC}_{90}$ was 0.19 $\mathrm{mg} / \mathrm{L}$. All 57 strains showed high susceptibility to both oxacillin and benzylpenicillin. However, MIC values $\leq 0.016 \mathrm{mg} / \mathrm{L}$ for benzylpenicillin and oxacillin were found in 51/57 (89.5\%) and 9/57 (15.8\%) of the isolates, respectively. The MIC values for benzylpenicillin were 3-4-fold lower when compared to oxacillin.

\section{Discussion}

Since $C$. acnes can be found in the sebaceous glands in human skin $[6,7,9,10]$ and is not eradicated by surface disinfection $[11,12,13]$, optimal prophylactic antibiotic treatment is of great importance during implant surgery. National guidelines from a Swedish national expert group recommend isoxazolylpenicillin (cloxacillin) as prophylactic antibiotic treatment for joint surgery patients in Sweden (PRISS Expert Group 2; http://lof.se/patientsakerhet/varaprojekt/rekommendationer/). This recommendation is indicated for knee and hip surgery, but hitherto has also been applied to shoulder implant surgery.

Due to the high number of shoulder PJIs caused by $C$. acnes, some departments and orthopedic surgeons have added benzylpenicillin to the isoxazolylpenicillin prophylactic treatment or replaced isoxazolyl-penicillin with clindamycin. This double prophylactic treatment is based on clinical experience, but no study on this subject has been published so far.

When comparing the MIC values of $C$. acnes obtained from implant-associated infections, we found that benzylpenicillin displayed 3-4-fold lower MIC values than isoxazolyl penicillin. These results indicate that benzylpenicillin should be evaluated as an addition to isoxazolyl-penicillin, if used as anti-staphylococcal prophylactic treatment for shoulder arthroplasty.

A higher dose of beta-lactam antibiotics is associated with an increased risk of adverse events such as gastrointestinal and nephrotoxicity. The risk of these complications should be evaluated and taken into consideration when changing prophylactic regimens. 


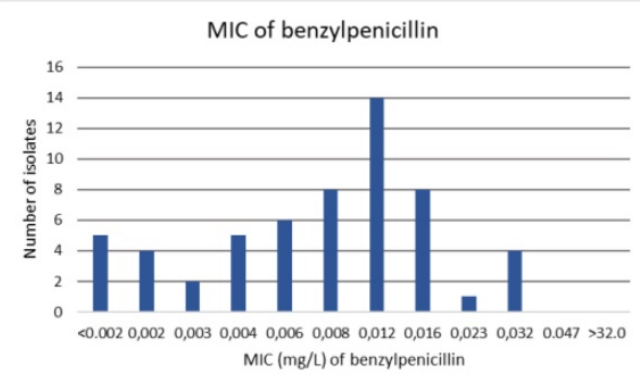

$1 \mathrm{a}$

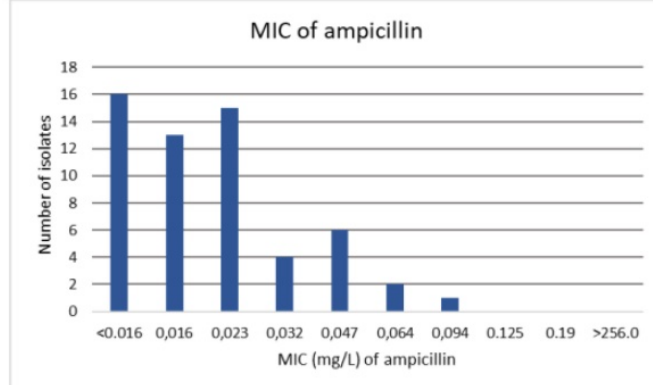

$1 c$

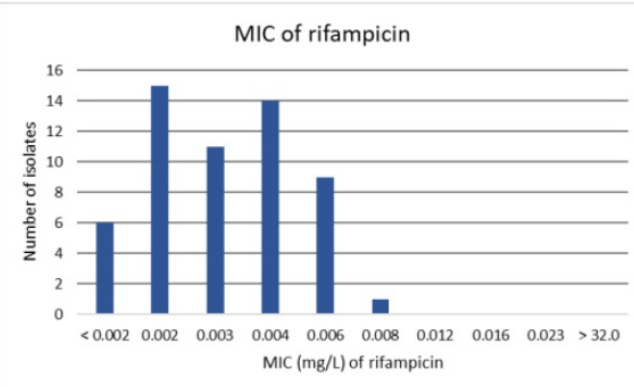

$1 e$

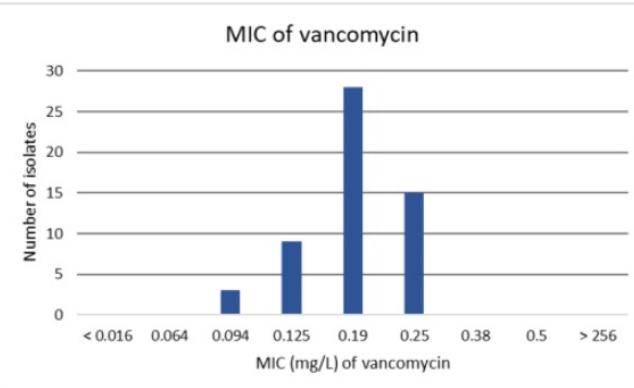

\section{$1 \mathrm{~g}$}

Figure 1. Distribution of MIC values determined by Etest for 57 isolates of Cutibacterium acnes obtained from orthopedic implant infections of the knee joint ( $\mathrm{n}=5$ ), hip joint $(n=21)$, and shoulder joint $(n=31)$ for; a) benzylpenicillin, b) oxacillin, c) ampicillin, d) cefuroxime, e) rifampicin, f) clindamycin, g) vancomycin, and $h$ ) daptomycin.

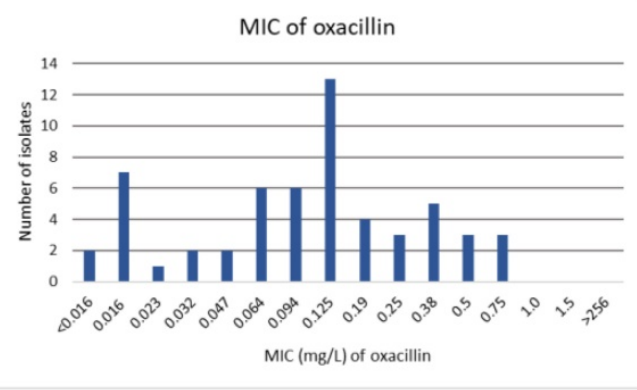

$1 b$

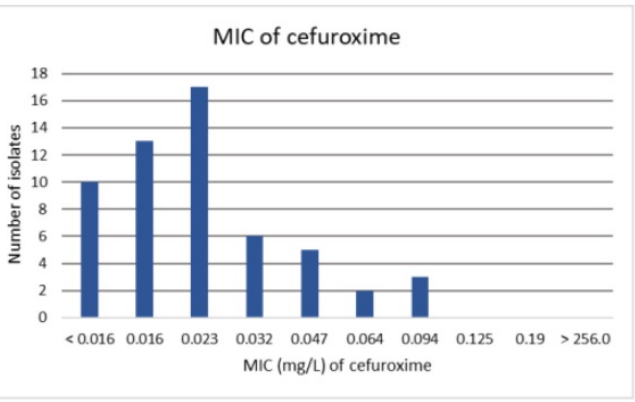

$1 d$

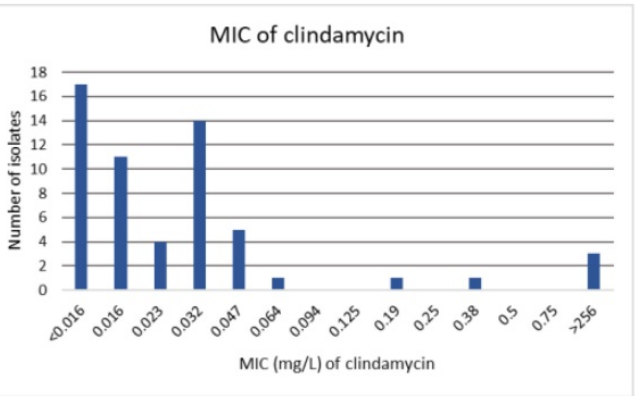

$1 f$

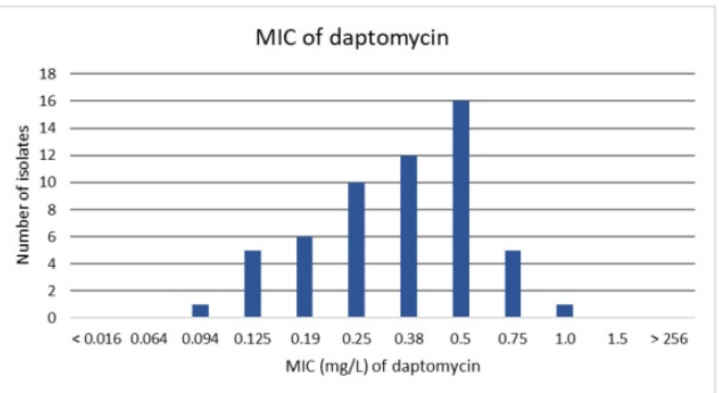

$1 \mathrm{~h}$ 


\section{Compared MIC values for benzylpenicillin and oxacillin}

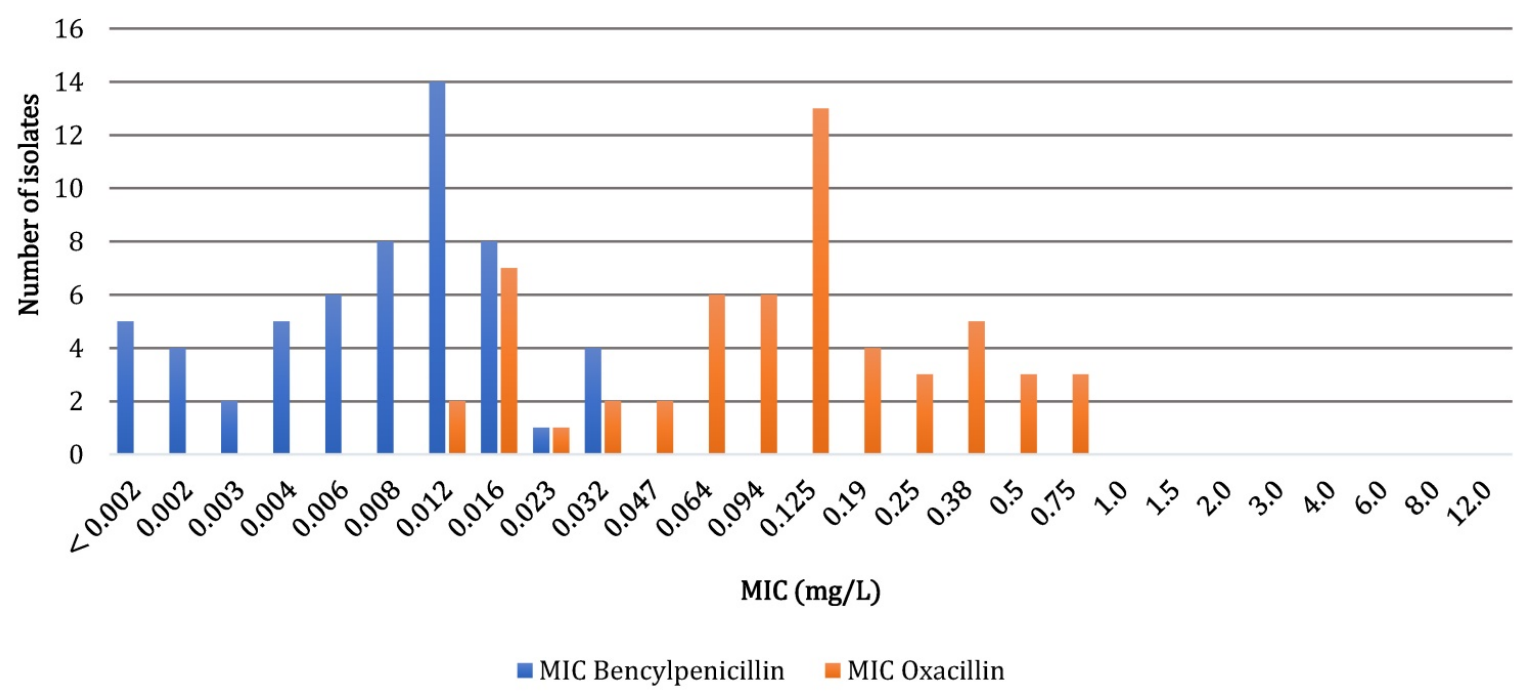

Figure 2. Comparison of MIC values determined by Etest for benzylpenicillin and oxacillin of 57 isolates of Cutibacterium acnes obtained from orthopedic implant infections. The lowest MIC values for benzylpenicillin are $<0.002 \mathrm{mg} / \mathrm{L}$ and those for oxacillin are $<0.016 \mathrm{mg} / \mathrm{l}$. Due to the different scales on the Etest strips, the lowest value for oxacillin ( $<0.016 \mathrm{mg} / \mathrm{L}$ ) is marked in the figure as $0.012 \mathrm{mg} / \mathrm{L}$. The real MIC values for these strains are assumed to be $0.012 \mathrm{mg} / \mathrm{L}$ or lower, but cannot be measured due to the scale on the Etest strips.

The clinical outcome of antimicrobial prophylaxis is not solely based only on the MIC value; other factors such as tissue penetration, pharmacokinetics and pharmacodynamics should also be considered [14]. Benzylpenicillin and isoxazolyl-penicillin are bactericidal antibiotics, which means that their effect is correlated with the length of time that the antibiotic concentration exceeds the MIC value. The half-life of benzylpenicillin is 30-50 minutes and the serum protein binding is approximately $65 \%$, while the half-life of cloxacillin is 30 minutes and the serum protein binding is $94-98 \%$. Since these antibiotics are bactericidal, the half-life is short, and the serum protein binding is high, the doses administered must be high and given repeatedly during the operating day to achieve an appropriate effect and maintain time above MIC.

All of the 57 C. acnes isolates investigated in this study had MIC values for benzylpenicillin of 0.032 $\mathrm{mg} / \mathrm{L}$ or lower. However, these are in vitro data, which is a limitation of the study. To evaluate the clinical effect of prophylactic benzylpenicillin, there is a great need for either register-based and/or controlled randomized clinical studies.

In the present study, MIC values were also determined for other antibiotic agents: ampicillin, cefuroxime, clindamycin, rifampicin, daptomycin, and vancomycin. Clindamycin was the only antibiotic agent with high MIC values for some isolates, with some exhibiting a high level of resistance. Similar results have been shown in previous studies $[8,12,15,16,19]$. This reinforces the fact that $C$. acnes can develop resistance against antibiotic agents.
Clindamycin is used as prophylactic treatment for patients with penicillin allergy. Furthermore, selected C. acnes isolates can develop resistance to clindamycin $[15,16,17,18,19]$. These factors should be taken into account when selecting clindamycin for surgical prophylaxis.

A limitation of the present study is that cefazolin was not assessed, since it is used as perioperative antimicrobial prophylaxis in many countries [2]. However, cefuroxime was assessed and was associated with low $\mathrm{MIC}_{50}$ and $\mathrm{MIC}_{90}$; the MIC values were 1 - 2 fold higher compared to benzylpenicillin. In addition, cephalosporins have a broader antimicrobial spectrum which may have negative ecological effects. The half-life of cephalosporins is longer when compared to penicillins, which affect the timing and frequency of administration [20].

The isolates investigated in the present study all showed low MIC values for rifampicin $(\leq 0.008 \mathrm{mg} / \mathrm{L})$, and none of the tested isolates were resistant. However, monotherapy with rifampicin usage can select for antibiotic resistance among skin isolates of staphylococci. Since staphylococci are the second most frequent pathogen in shoulder PJIs, rifampicin monotherapy should not be used in a prophylaxis, but rather be reserved as a treatment option for an established PJI. It has also been shown that C. acnes has the ability to develop resistance against rifampicin in the same manner as staphylococci; that is, by specific point mutations, which again makes rifampicin monotherapy an inappropriate prophylactic treatment [21].

According to EUCAST (eucast.org), the only 
specific MIC breakpoints (epidemiological cut-off values) noted for $C$. acnes are $0.125 \mathrm{mg} / \mathrm{L}$ for benzylpenicillin and $2.0 \mathrm{mg} / \mathrm{L}$ for vancomycin. Daptomycin could also be considered as a prophylactic agent, since it is active against staphylococci including multi-drug resistant Staphylococcus epidermidis. However, as of yet there are no reports on the use of daptomycin for prophylaxis in implant surgery.

\section{Conclusion}

The MIC values for benzylpenicillin were 3-4-fold lower when compared to cloxacillin. These in vitro results indicate that benzylpenicillin might be a more effective drug to prevent shoulder PJIs caused by $C$. acnes, However, further studies on the subject are needed, and the efficiency and safety of the prophylactic combination therapy should be evaluated using a randomized controlled clinical trial and/or register-based studies.

\section{Acknowledgements}

This study was supported by Nyckelfonden at Örebro University Hospital (OLL-5955951).

\section{Competing Interests}

The authors have declared that no competing interest exists.

\section{References}

1. AlBuhairan B, Hind D, Hutchinson A. Antibiotic prophylaxis for wound infections in total joint arthroplasty: a systematic review. J Bone Joint Surg Br 2008; 90(7): 915-9.

2. Tande AJ, Patel R. Prosthetic joint infection. Clin Microbiol Rev 2014; 27(2): 302-45.

3. Zimmerli W, Trampuz A, Ochsner PE. Prosthetic-joint infections. N Engl J Med 2004; 351(16): 1645-54

4. Levy PY, Fenollar F, Stein A, et al. Propionibacterium acnes postoperative shoulder arthritis: an emerging clinical entity. Clin Infect Dis 2008; 46(12): 1884-6.

5. Lutz MF, Berthelot P, Fresard A, et al. Arthroplastic and osteosynthetic infections due to Propionibacterium acnes: a retrospective study of 52 cases, 1995-2002. Eur J Clin Microbiol Infect Dis 2005; 24(11): 739-44.

6. Portillo ME, Corvec S, Borens $\mathrm{O}$, et al. Propionibacterium acnes: an underestimated pathogen in implant-associated infections. Biomed Res Int 2013; 2013: 804391

7. Achermann Y, Goldstein EJ, Coenye T, et al. Propionibacterium acnes: from commensal to opportunistic biofilm-associated implant pathogen. Clin Microbiol Rev 2014; 27(3): 419-40.

8. Kadler BK, Mehta SS, Funk L. Propionibacterium acnes infection after shoulder surgery. Int J Shoulder Surg 2015; 9(4): 139-44.

9. Bojar RA, Holland KT. Acne and Propionibacterium acnes. Clin Dermatol 2004; 22(5): 375-9.

10. Perry AL, Lambert PA. Propionibacterium acnes. Lett Appl Microbiol 2006; 42(3): 185-8.

11. Falk-Brynhildsen $\mathrm{K}$, Söderquist $\mathrm{B}$, Friberg $\mathrm{O}$, et al. Bacterial recolonization of the skin and wound contamination during cardiac surgery: a randomized controlled trial of the use of plastic adhesive drape compared with bare skin. J Hosp Infect 2013; 84(2): 151-8.

12. Phadnis J, Gordon D, Krishnan J, et al. Frequent isolation of Propionibacterium acnes from the shoulder dermis despite skin preparation and prophylactic antibiotics. J Shoulder Elbow Surg 2016; 25(2): 304-10.

13. Matsen FA 3rd, Russ SM, Bertelsen A, et al Propionibacterium can be isolated from deep cultures obtained at primary arthroplasty despite intravenous antimicrobial prophylaxis. J Shoulder Elbow Surg. 2015; 24(6): 844-7.

14. Mouton JW, Brown DFJ, Apfalter $\mathrm{P}$, et al. The role of pharmacokinetics/pharmacodynamics in setting clinical MIC breakpoints: the EUCAST approach. Clin Microbiol Infect 2012; 18(3): E37-45.
15. Khassebaf J, Hellmark B, Davidsson S, et al. Antibiotic susceptibility of Propionibacterium acnes isolated from orthopaedic implant-associated infections. Anaerobe 2015; 32: 57-62.

16. Crane JK, Hohman DW, Nodzo SR, et al. Antimicrobial susceptibility of Propionibacterium acnes isolates from shoulder surgery. Antimicrob Agents Chemother 2013; 57(7): 3424-6.

17. Oprica C, Nord CE, ESCMID Study Group on Antimicrobial Resistance in Anaerobic Bacteria. European surveillance study on the antibiotic susceptibility of Propionibacterium acnes. Clin Microbiol Infect 2005; 11(3): 204-13.

18. Walsh TR, Efthimiou J, Dréno B. Systematic review of antibiotic resistance in acne: an increasing topical and oral threat. Lancet Infect Dis 2016; 16(3): e22-32.

19. Wright TE, Boyle KK, Duquin TR, Crane JK. Propionibacterium acnes Susceptibility and Correlation with Hemolytic Phenotype. Infect Dis (Auckl). 2016; 9: 39-44.

20. Stefánsdóttir A, Robertsson $\mathrm{O}, \mathrm{W}$-Dahl A, et al. Inadequate timing of prophylactic antibiotics in orthopedic surgery. We can do better. Acta Orthop. 2009; 80(6): 633-8

21. Furustrand Tafin U, Trampuz A, Corvec S. In vitro emergence of rifampicin resistance in Propionibacterium acnes and molecular characterization of mutations in the rpoB gene. J Antimicrob Chemother 2013; 68(3): 523-8. 